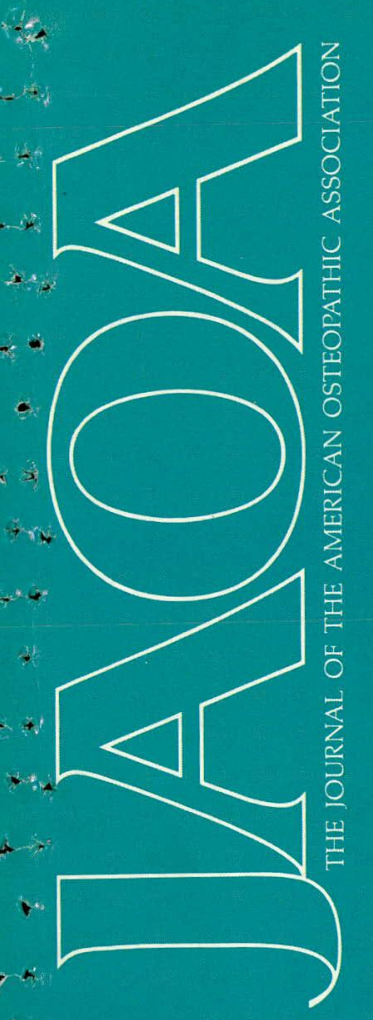

MARCH 1986

863

Surface pattern of stomach and duodenum in a chronic renal failure cohort

Childhood diskitis

\title{
Central venous
} catheterization infection

CME quiz
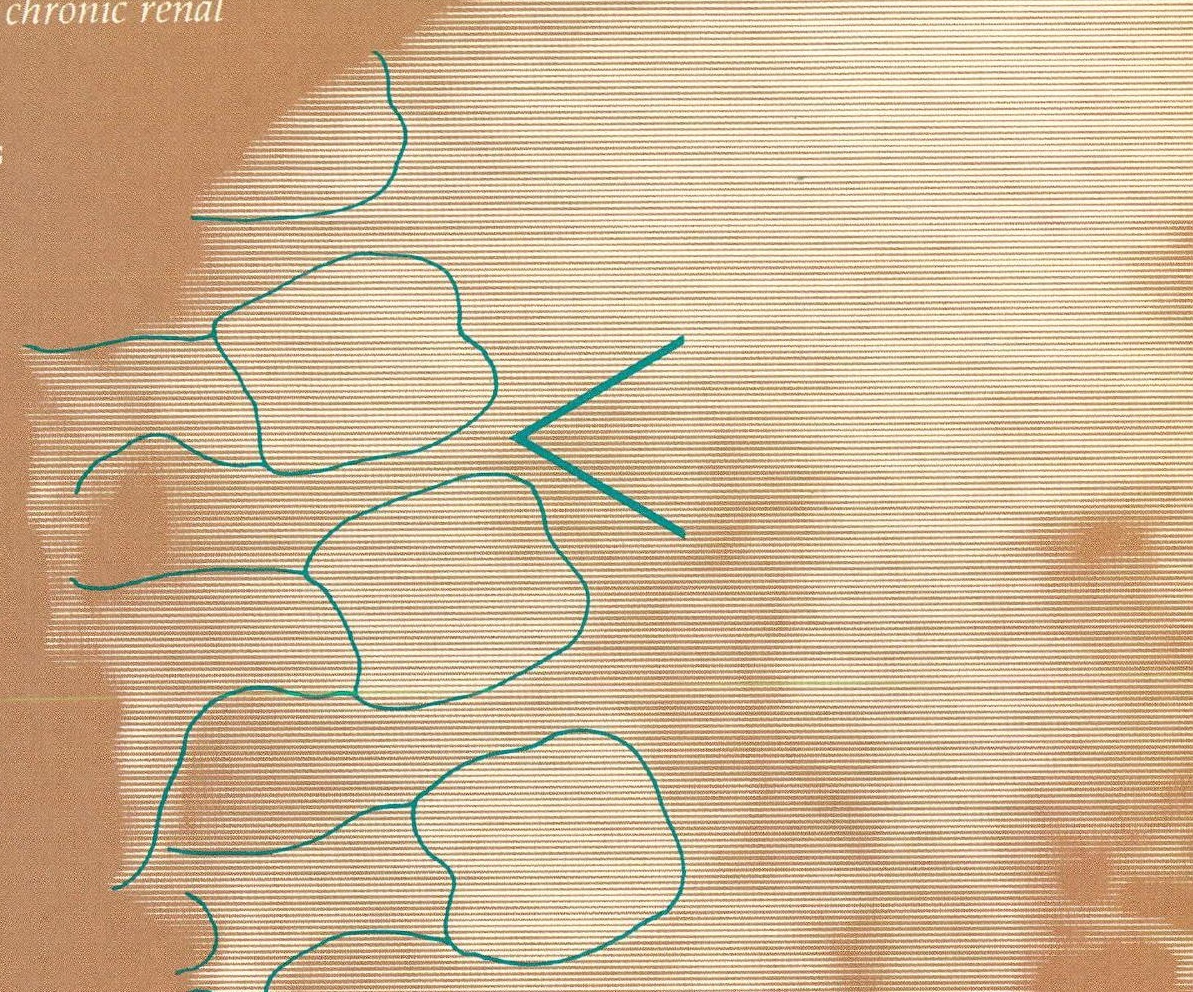

(
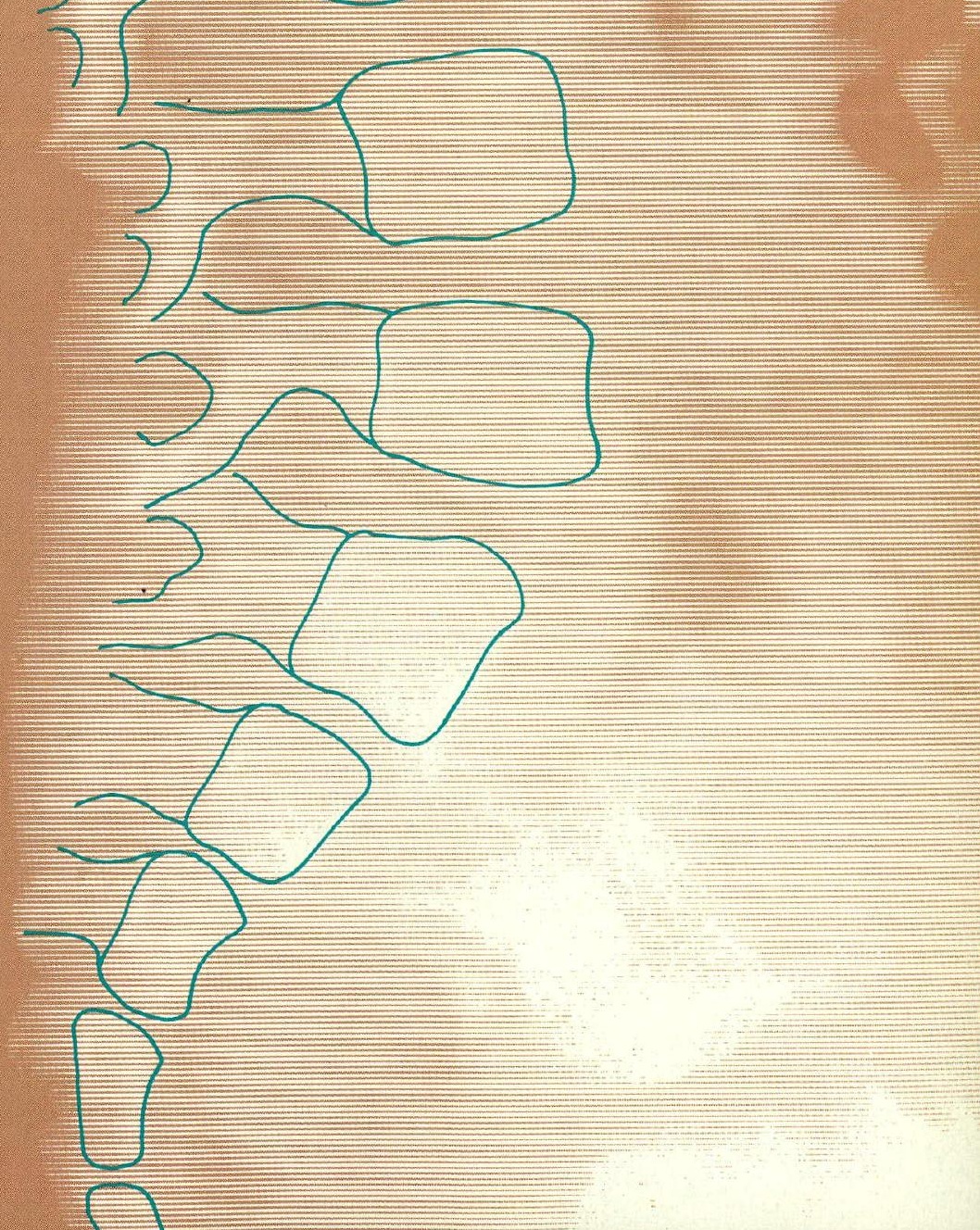


\section{Will the doctors of tomorrow}

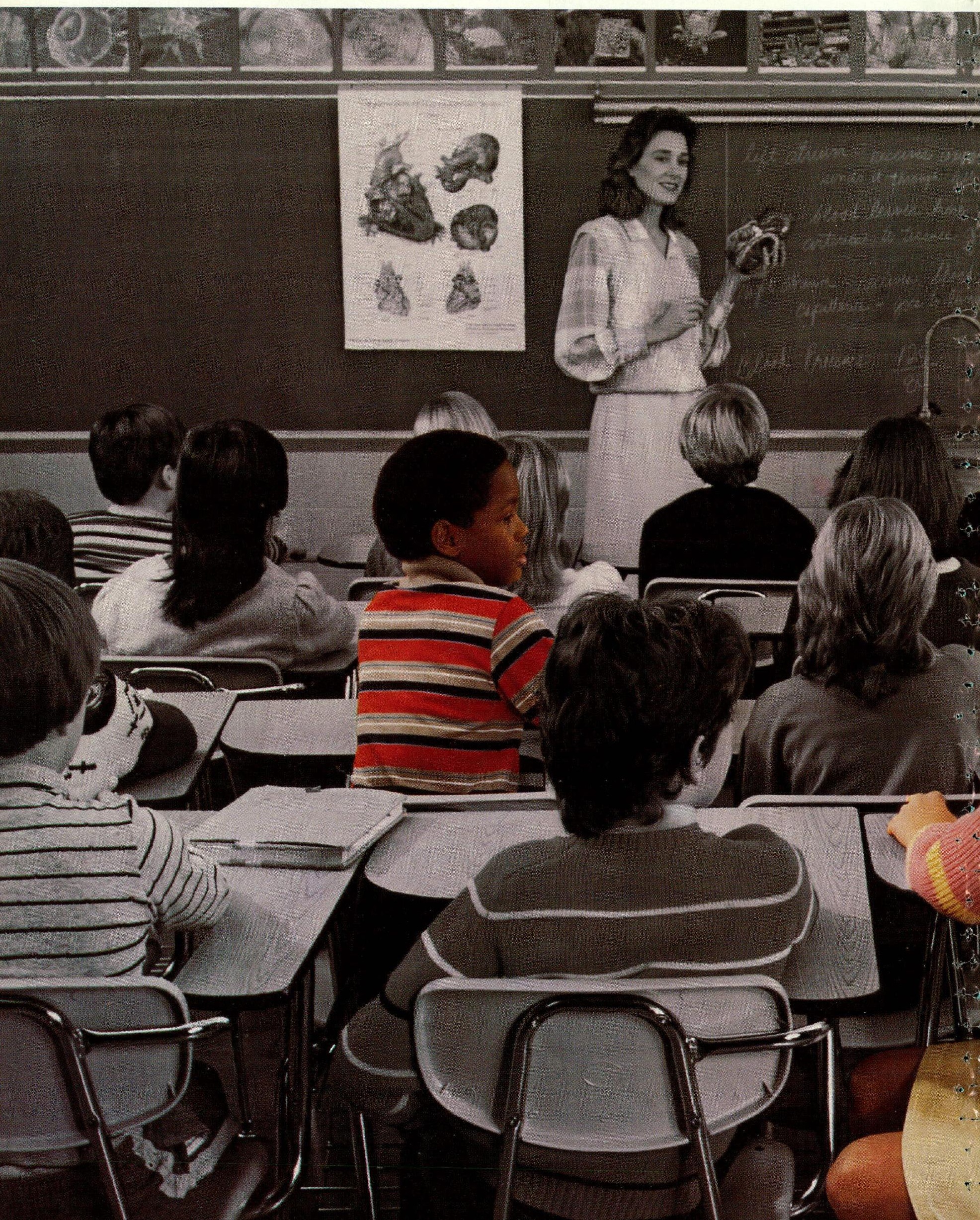


Only if manufacturers of brand-name pharmaceuticals like Roche can continue to develop new products at a time when making them available is both unpredictable and expensive.

On the average, only one in 10,000 promising new compounds is ever introduced as a new product. And about $\$ 90$ million is usually spent on that product before it's available for your prescription. To follow up on discoveries like Valium ${ }^{\circledast}$ (brand of diazepam/Roche), Roche is now spending, on a worldwide basis, about 20 cents of every sales dollar in up-front drug research and development. It's our way of making sure that tomorrow's drugs are available tomorrow.

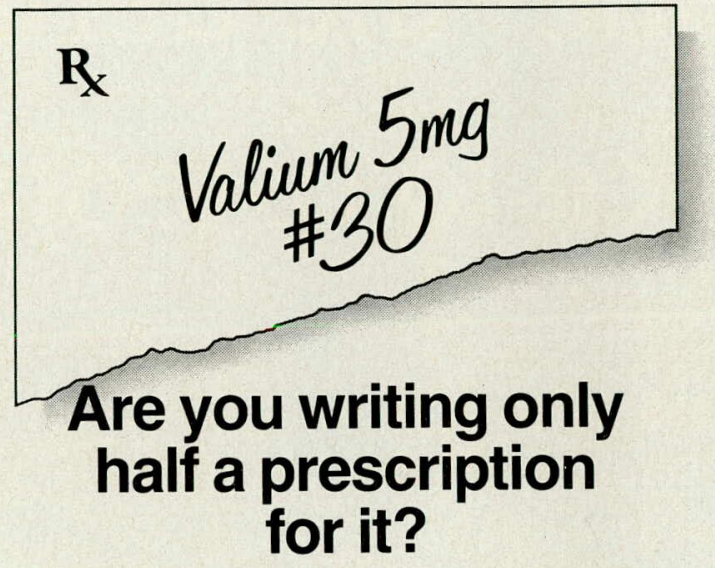

This half is important too!

Be sure to write a complete prescription.

Specify "Dispense as written" or

"Do not substitute" or "Medically necessary."

\section{Do Not Substitute}

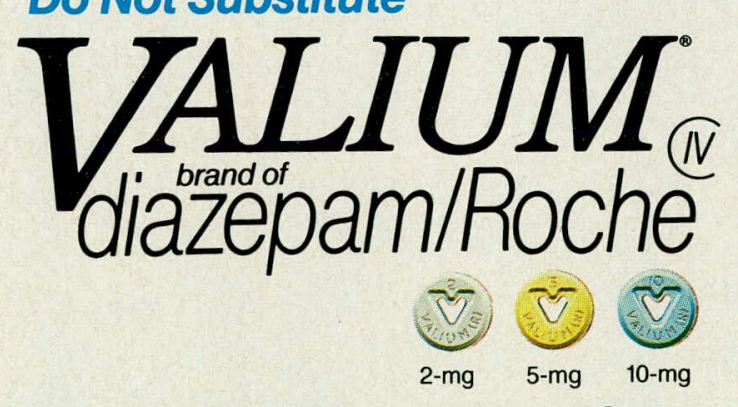

Confidence through Experience

The cut out " $V$ " design is a registered trademark of Roche Products Inc. 


\section{olio $|\bar{o}-\mathrm{e}-\overline{\mathrm{e}}, \overline{\mathrm{o}}|$ $\mid$ 1: a miscellaneous}

\section{collection of literary selections}

\section{2: Osteopathic Literature Index/}

Online-the definitive online

\section{source to osteopathic literature}

Osteopathic Literature Index/Online

Now available through $\square \square$

For further information, contact AONET,

the American Osteopathic Network,

1500 Walnut Street, 7 th floor,

Philadelphia, PA 19102

(215) $875-4650$ or (800) 332-ICOA 


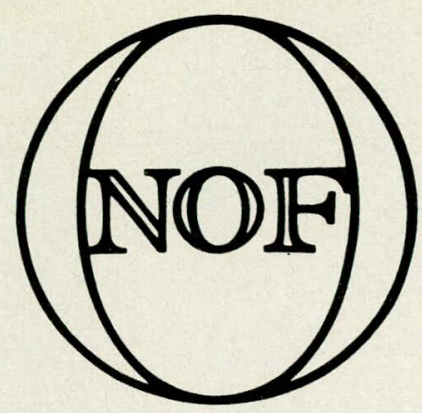

\section{Give Your Patients A Chance...}

to participate in the good works of your profession...

to provide urgently needed funds for loans to students in your colleges...

to support important research in your institutions

\section{Osteopathic Seals Support... Student Loans, Osteopathic Research}

\section{Use Osteopathic Seals.}

Your own contribution is important but the gifts of your patients, friends, those with whom you do business, represent by far the greatest potential...

and you can give them information on the profession and secure their involvement at the same time...

read the Seals material when it arrives... order packets and mail them.. display them in your office...

\section{Give Your Patients}
A Chance...
Use Osteopathic Seals.

Osteopathic Seal Program 212 East Ohio Street Chicago, Illinois 60611
KAON CL-10 ${ }^{\text {TM }}$ (potassium chloride) Controlled-Release Tablets

KAON-CL ${ }^{\circledast}$ (potassium chloride) Controlled-Release Tablets

Description: KAON CL-10 is a sugar coated (not enteric-coated) tablet containing $750 \mathrm{mg}$ potassium chloride (equivalent to $10 \mathrm{mEq}$ potassium chloride) in a wax matrix. This formulation is intended to provide a controlled release of potassium from the matrix to minimize the likelihood of producing high localized concentrations of potassium within the gastrointestinal tracl

KAON-CL is a sugar coated (not enteric-coated) tablet containing $500 \mathrm{mg}$ potassium chloride (equivalent to 6.7 mEq potassium chloride) in a wax matrix. Contains color additives including FD\&C Yellow No. 5 (tartrazine). This formulation is intended to provide a controlled release of potassium from the matrix to minimize the likelihood of producing high localized concentrations of potassium within the gastrointestinal tract.

Indications:

BECAUSE OF REPORTS OF INTESTINAL AND GASTRIC ULCERATION AND BLEEDING WITH SLOW RELEASE POTASSIUM CHLORIDE PREPARATIONS, THESE DRUGS SHOULD BE RESERVED FOR THOSE PATIENTS WHO CANNOT TOLERATE OR REFUSE TO TAKE LIQUIDS OR EFFERVESCENT POTASSIUM PREPARATIONS OR FOR PATIENTS IN WHOM THERE IS A PROBLEM OF COMPLIANCE WITH THESE PREPARATIONS.

1. For therapeutic use in patients with hypokalemia with or without metabolic alkalosis, in digitalis intoxication and in patients with hypokalemic familial periodic paralysis.

For the prevention of potassium depletion when the dietary intake is inadequate in the following conditions: Patients receiving digitalis and diuretics for congestive heart failure; hepatic cirrhosis with ascites, states of aldosterone excess with normal renal function, potassium-losing nephropathy and with certain diarrheal states The use of potassium salts in patients receiving diuretics for uncomplicated essential hypertension is often unnecessary when such patients have a normal dietary pattern. Serum potassium should be checked periodcally, however, and if hypokalemia occurs, dietary supplementation with potassium-containing foods may be adequate to control milder cases. In more severe cases supplementation with potassium salts may be indicated Contraindications: Potassium supplements are contraindicated in patients with hyperkalemia since a further increase in serum potassium concentration in such patients can produce cardiac arrest. Hyperkalemia may complicate any of the following conditions: Chronic renal failure systemic acidosis such as diabetic acidosis, acute dehydration, extensive tissue breakdown as in severe burns, adrenal insufficiency, or the administration of a potassium-sparing diuretic (e.g. spironolactone triamterene).

potassium-sparing diuretic (e.g., spironolactone, triamterene). with with esophageal compression chould be with a liquid preparation.

All solid dosage forms of potassium chloride supplements are contraindicated in any patient in whom there is cause for arrest or delay in tablet passage through the gastrointestinal tract. In these instances, potassium supplementation should be with a liquid preparation.

Warnings:

Hyperkalemia - In patients with impaired mechanisms for excreting potassium, the administration of potassium salts can produce hyperkalemia and cardiac arrest. This occurs most commonly in patients given potassium by the intravenous route but may also occur in patients given potassium orally. Potentially fatal hyperkalemia can develop rapidly and be asymptomatic. The use of potassium salts in patients with chronic renal disease, or any other condition which impairs potassium excretion, requires particularly careful monitoring of the serum potassium concentration and appropriate dosage adjustment.

Interaction with Potassium Sparing Diuretics - Hypokalemia should not be treated by the concomitant administration of potassium salts and a potassium-sparing diuretic (e.g., spironolactone or triamterene) since the simutaneous administration of these agents can produce severe hyperkalemia.

Gastrointestinal Lesions - Potassium chloride tablets have produced stenotic and/or ulcerative lesions of the small bowel and deaths. These lesions are caused by a high localized concentration of potassium ion in the region of a rapidly dissolving tablet which injures the bowel wall and thereby produces obstruction, hemorrhage, or perforation. KAON-CL and KAON CL-10 are wax-matrix tablets formulated to provide a controlled rate of release 0 perssium. polassium chiloride and thus to minimize the possibixy la io is bowel wall. While he reported frequency of small bowel lesions is mch labs ner 100,000 palint

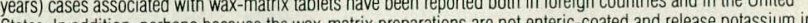
States. In addition, perhaps because the wax-matrix preparations are not enteric-coated and release potassium in the stomach, there have been reports of upper gastrointestinal bleeding associated with these products. The total number of gastrointestinal lesions remains less than one per bo, 000 palient years. KAON-CL and KAON CL- 0 should be discontinued immediately and the possibility of bowel obstruction or perforation considered if severe
vomiting, abdominal pain, distention, or gastrointestinal bleeding occurs.

Metabolic Acidosis - Hypokalemia in patients with metabolic acidosis should be treated with an alkalinizing potassium salt such as potassium bicarbonate, potassium citrate, potassium acetate, or potassium gluconate. Precautions: The diagnosis of potassium depletion is ordinarily made by demonstrating hypokalemia in a patient with a clinical history suggesting some cause for potassium depletion. In interpreting the serum potassium level, the physician should bear in mind that acute alkalosis per se can produce hypokalemia in the absence of a defici in total body potassium while acute acidosis per se can increase the serum potassium concentration into the normal range even in the presence of a reduced total body potassium. The treatment of potassium depletion,

particularly in the presence of cardiac disease, renal disease, or acidosis requires careful attention to acid-base particularty in the clinical status of the balance an

KAON-CL contains FD\&C Yellow No 5 (tartrazine) which may cause allergic-type reactions (including bronCon sensitivity in the general population is low, it is frequently seen in patients who also have aspirin hypersensitivity Adverse Reactions: The most common adverse reactions to oral potassium salts are nausea, vomiting, abdominal discomfort and diarrhea. These symptoms are due to irritation of the gastrointestinal tract and are best managed by diluting the preparation further, taking the dose with meals, or reducing the dose.

The most severe adverse effects are hyperkalemia (see Contraindications, Warnings and Overdosage) and gastrointestinal obstruction, bleeding or perforation (see Warnings).

Overdosage: The administration of oral potassium salts to persons with normal excretory mechanisms for Overdosage: The administration or oral polassium salls to excretory mechanisms are impaired or if potassium is administered too rapidly intravenously potentially fatal hyperkalemia can result (see Contraindications and Warn-

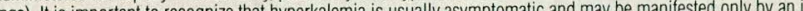
ings). It is important to recognize that hyperkalemia is ustally asymplomalic and may be manesesto only by increased ser pression of S-T segnt, and prolongation of the QT interval). Late manifestations include muscleparalysis and cardiovascular collapse from cardiac arrest.

Treatment measures for hyperkalemia include the following:

1. Flimination of foods and medications containing potassium and of potassium-sparing diuretics.

2. Intravenous administration of 300 to $500 \mathrm{ml} / \mathrm{hr}$ of $10 \%$ dextrose solution containing $10-20$ units of crystalline insulin per $1,000 \mathrm{ml}$.

3 Correction of acidosis, if present, with intravenous sodium bicarbonate

4. Use of exchange resins, hemodialysis, or peritoneal dialysis.

In treating hyperkalemia, it should be recalled that in patients who have been stabilized on digitalis, too rapid a lowering of the serum potassium concentration can produce digitalis toxicity.

CAUTION: Federal law prohibits dispensing without prescription.

HOW SUPPUIED: KAON CL-10'm is supplied in bottles of 100 and 500. STAT-PAK ${ }^{\circledR}$ (unit dose) box of 100. KAON-C ${ }^{\oplus}$ is supplied in bottles of 100,250 , and 1000 .

For prevention or treatment of hypokalemia when a non-liquid potassium chloride is indicated.

There have been no reported cases of small bowel stenosis or ulceration in over ten years of clinical experience and one billion doses of KAON-CL and KAON CL-10. Reported frequency of Gl lesions with wax matrix tablets is less than one per 100,000 patient years. 


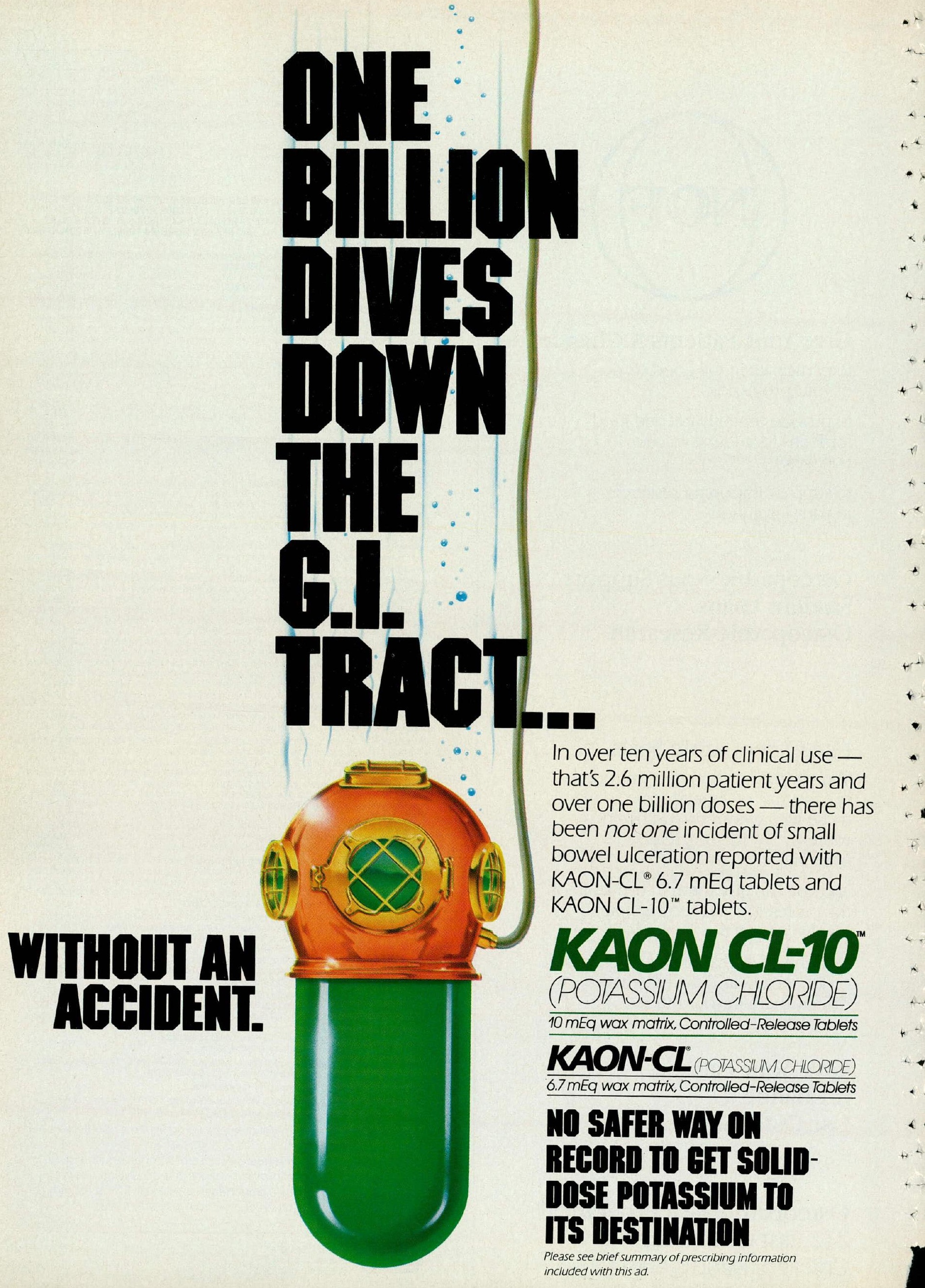

